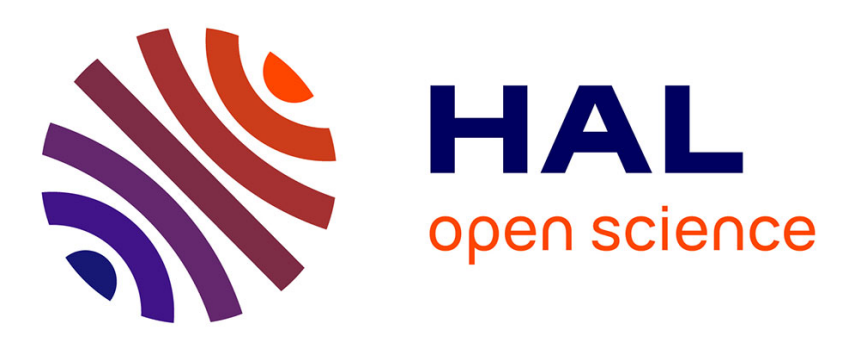

\title{
A "top-down" in silico approach for designing ad hoc bio-based solvents: application to glycerol-derived solvents of nitrocellulose
}

Laurianne Moity, Valérie Molinier, Adrien Benazzouz, Benjamin Joossen, Vincent Gerbaud, Jean-Marie Aubry

\section{To cite this version:}

Laurianne Moity, Valérie Molinier, Adrien Benazzouz, Benjamin Joossen, Vincent Gerbaud, et al.. A "top-down" in silico approach for designing ad hoc bio-based solvents: application to glycerol-derived solvents of nitrocellulose. Green Chemistry, 2016, 18 (11), pp.3239-3249. 10.1039/C6GC00112B . hal-01340167

\section{HAL Id: hal-01340167 \\ https://hal.science/hal-01340167}

Submitted on 30 Jun 2016

HAL is a multi-disciplinary open access archive for the deposit and dissemination of scientific research documents, whether they are published or not. The documents may come from teaching and research institutions in France or abroad, or from public or private research centers.
L'archive ouverte pluridisciplinaire HAL, est destinée au dépôt et à la diffusion de documents scientifiques de niveau recherche, publiés ou non, émanant des établissements d'enseignement et de recherche français ou étrangers, des laboratoires publics ou privés. 


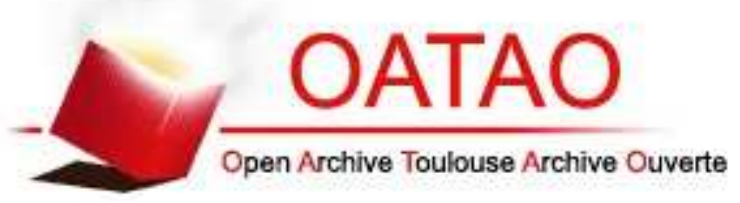

\section{Open Archive TOULOUSE Archive Ouverte (OATAO)}

OATAO is an open access repository that collects the work of Toulouse researchers and makes it freely available over the web where possible.

This is an author-deposited version published in : http://oatao.univ-toulouse.fr/ Eprints ID : 15878

To link to this article : DOI : 10.1039/C6GC00112B

URL : http://dx.doi.org/10.1039/C6GC00112B

To cite this version : Moity, Laurianne and Molinier, Valérie and Benazzouz, Adrien and Joossen, Benjamin and Gerbaud, Vincent and Aubry, Jean-Marie A "top-down" in silico approach for designing ad hoc bio-based solvents: application to glycerol-derived solvents of nitrocellulose. (2016) Green Chemistry, vol. 18 ( $\left.\mathrm{n}^{\circ} 11\right)$. pp. 3239-3249. ISSN 1463-9262

Any correspondence concerning this service should be sent to the repository administrator: staff-oatao@ listes-diff.inp-toulouse.fr 


\title{
A "top-down" in silico approach for designing ad hoc bio-based solvents: application to glycerol-derived solvents of nitrocellulose
}

\author{
Laurianne Moity, ${ }^{a}$ Valérie Molinier, ${ }^{\star a}$ Adrien Benazzouz, ${ }^{a}$ Benjamin Joossen, ${ }^{a}$ \\ Vincent Gerbaud ${ }^{b}$ and Jean-Marie Aubry ${ }^{\star a}$
}

\begin{abstract}
Potentially effective glycerol-based solvents for nitrocellulose have been designed using a top-down in silico procedure that combines Computer Assisted Organic Synthesis (CAOS) and Molecular Design (CAMD). Starting from a bio-based building block - glycerol - a large number of synthetically feasible chemical structures have been designed using the GRASS (GeneratoR of Agro-based Sustainable Solvents) program. GRASS applies well-selected industrial chemical transformations to glycerol together with a limited number of relevant co-reactants. Then, the most promising structures are considered as lead compounds for further modification in silico thanks to the IBSS (InBioSynSolv) program, which generates derivatives with alkyl, cycloalkyl, alkene, cycloalkene or phenyl substituents. Finally, IBSS ranks all the candidates according to the value of their overall performance function to best fit the predefined specifications, i.e. (i) high solubilisation of nitrocellulose, (ii) slow evaporation and non-flammability (iii) low toxicity and environmental impact. This general strategy enables the highlighting of the most relevant solvent candidate derived from any building block for a given application. To validate the approach, 15 commercially available solvents derived from glycerol were confronted with nitrocellulose and led to highlight diacetin as an effective and safe solvent.
\end{abstract}

\section{Introduction}

The search for alternative solvents is a topic of major interest from both academic and industrial points of view. ${ }^{1-4}$ In some applications, especially those without appropriate safety precautions for consumers, finding alternatives to harmful traditional solvents has become mandatory. This is the case for instance for paint removing, degreasing or coating formulations that traditionally used a plethora of solvents with a poor environmental footprint, such as halogenated, aromatic or glycol derivatives, which are now banned. For these formulations, in which the role of solvent is crucial and involves many different physical and chemical properties, an efficient substitution methodology is required to find the best alternative candidate(s). Among the so-called "green" solvents, which are non-toxic and have a good environmental footprint, the bio-based ones are prepared from molecules readily available from the biomass feedstock. ${ }^{5-7}$ Despite a dynamic and renewable supply of bio-based chemicals, there is a need to develop

${ }^{a}$ Univ. Lille, CNRS, Centrale Lille, ENSCL, Univ. Artois, UMR 8181 - UCCS - Unité de Catalyse et Chimie du Solide, F-5900o Lille, France.

E-mail: jean-marie.aubry@ensc-lille.fr, valerie.molinier@ensc-lille.fr

${ }^{b}$ Université de Toulouse, INP, CNRS, LGC (Laboratoire de Génie Chimique), ENSIACET, 4 Allée Emile Monso, F-31432 Toulouse, France new solvent structures to match specific properties, which should be guided by efficient in silico tools. ${ }^{8-10}$

We have recently developed a Computer Assisted Organic Synthesis (CAOS) program named GRASS, standing for GeneratoR of Agro-based Sustainable Solvents. This program generates structures from a chosen bio-based building block by applying industrially relevant transformations to any starting molecule. ${ }^{11}$ The relevance of this tool has been exemplified starting from an emerging building block, itaconic acid, from which several families of potential solvents have been highlighted. The strength of this tool is that all the structures generated are supposed to be experimentally feasible, since they are obtained through well-selected chemical transformations. However, as GRASS requires the choice of co-reactants, the structures obtained should only be regarded as "lead compounds" that may not be the best candidate within a family to match the required properties. Also, only the first generation compounds can reasonably be examined because of the combinatorial explosion afterwards and the lack of properties calculation tools to sort out the molecules.

Another approach to generate virtual structures of potential solvents is the IBSS (InBioSynSolv) program. IBSS is a Computer Aided Product Design (CAPD) tool that defines a set of target values for selected properties and search for new molecules - individual or mixtures - that best satisfy them. The 
structures are optimized during the search thanks to a genetic algorithm and only the best candidates are proposed. ${ }^{12}$ IBSS can generate structures "from scratch" or starting from selected building blocks, including ones coming from the biomass feedstock. ${ }^{13}$ A drawback of this approach is that no safeguard ensures the viability of the generated structures. Therefore, the best structures proposed might be virtually excellent but practically non-feasible at an industrial scale.

In the present work, we have taken advantage of both approaches by combining the ability of GRASS to generate rational structures and the potentialities of IBSS to predict properties and to rank and optimize molecules thanks to a performance function. Here, IBSS was allowed to perform only slight structural modifications to the alkyl substituents introduced by GRASS at the first generation - chain length increase or branching, introduction of unsaturations or phenyl groups - to match the performances defined in the specifications.

To highlight this strategy, we have chosen the design of glycerol-based solvents for nitrocellulose as a relevant case. Nitrocelluloses with a low substitution degree are used for coatings, especially for nail varnishes and wood lacquers. In these formulations, the polymer is dissolved in a solvent - or a mixture of solvents - that allows low-viscosity formulations, easy spreading and formation of a glossy film after evaporation. In nitrocellulose lacquers, the solvents used are referred to as "lacquer thinners" and are usually mixtures of butyl acetate and aromatic solvents (xylene, toluene), which are all flammable and for the latter two, exhibit risks for human safety. This is therefore a good example of solvent substitution. We have chosen to look for a solvent derived from glycerol, which is an archetypical building block from the biomass feedstock as it received considerable interest in the last decade. ${ }^{14-17}$
Several solvents derived from glycerol are already described in the literature or available on the market, especially glycerol esters, glycerol carbonate and glycerol acetals. ${ }^{18-23}$

The specifications here are for the solvent to exhibit high solubilisation capacity towards nitrocellulose, high flash point to be considered as "non-flammable" and middle-range boiling point for slow evaporation ensuring good film formation and levelling. Additional prediction methods regarding EHS (Environmental, Health, Safety) profile are also included in the evaluation. Finally, to validate the approach, 15 commercially available solvents derived from glycerol were confronted with nitrocellulose to search for effective and safe solvents able to replace conventional solvents.

\section{Results and discussion}

Top-down approach used to design the most promising bio-solvents for a given solute

We are using here a "top-down" approach to propose promising solvents for a given solute, which means that we first define the properties that the solvent should comply with and we then generate in silico the structures best fitting the specifications following the three-step process depicted in Fig. 1. The final goal is to point out the most promising bio-based solvents satisfying at the same time technical and EHS-related properties.

In the first step, the target solute is chosen. Here, the methodology is illustrated with the example of a highly polar polymer, nitrocellulose, for which the behaviour against classical solvents is well-described by the Hansen solubility parameters approach. ${ }^{24}$ Specifications related to the solubilisation of nitro-

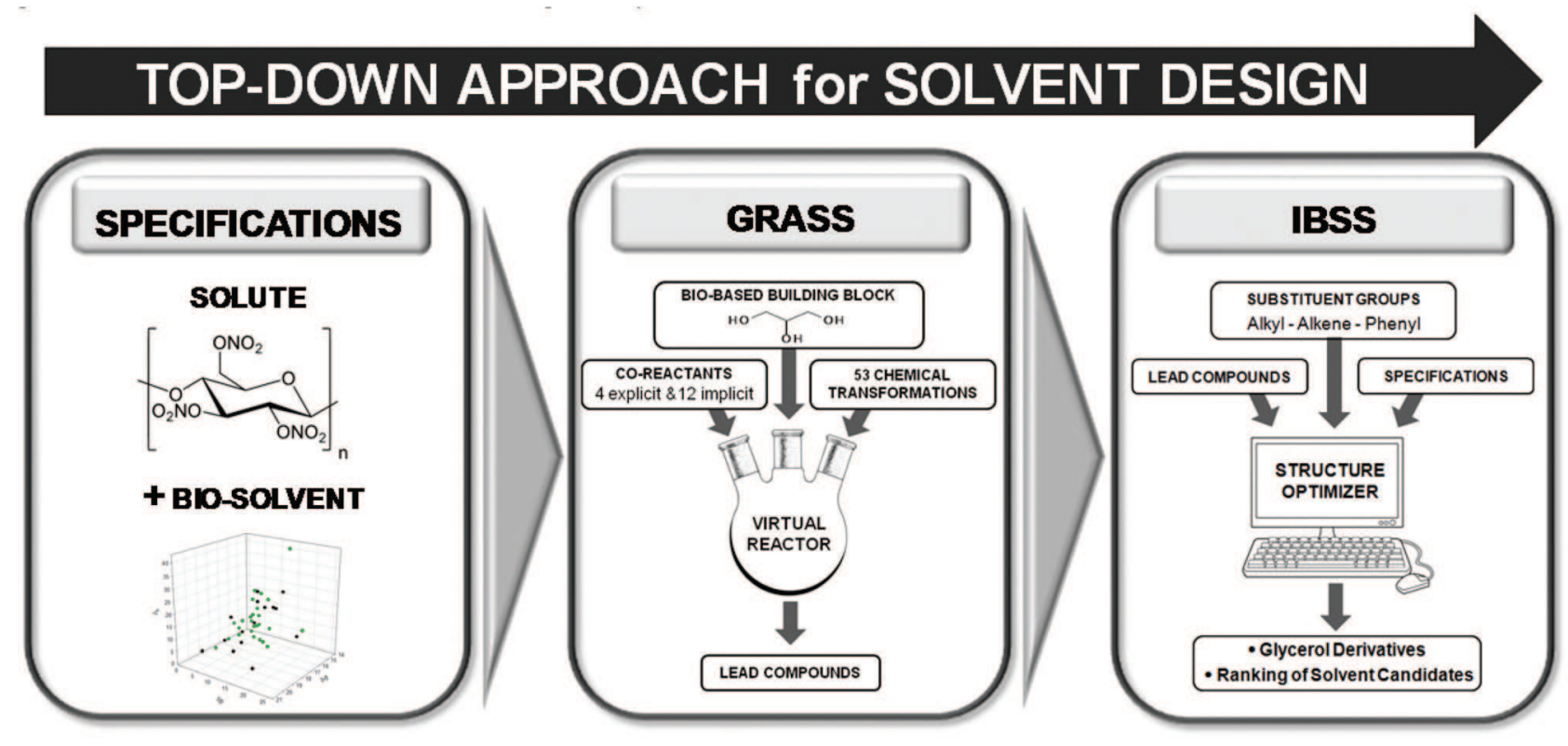

Fig. 1 Schematic representation of the top-down strategy used to find out in silico the most promising glycerol-derived solvents for nitrocellulose using GRASS and IBSS softwares. 
cellulose are therefore based on its so-called Hansen solubility sphere, as described in the next section. Additional technical and EHS-related specifications are also defined at this first step.

In the second step, plausible generic structures derived from a given molecule are generated thanks to a home-made software called GRASS for GeneratoR of Agro-based Sustainable Solvents. ${ }^{11}$ A readily available petro- or bio-based starting building block is introduced into a virtual reactor in which it can undergo 53 industrially-relevant chemical transformations in the presence of a limited number of simple co-reactants. Here, an archetypical starting molecule, i.e. glycerol, is chosen. The generic molecules obtained at this stage are industrially plausible and represent "lead compounds" that serve as inputs for the third step.

In the last step, the previously developed IBSS (InBioSynSolv) software ${ }^{12}$ has a twofold use: to generate a large number of simple derivatives from the "lead compounds" generated by GRASS and to rank all candidates according to their compliance with the specifications. The list of ranked molecules may contain already known solvents or new structures. It should be carefully examined in the eye of an organic chemist in order to select the most accessible candidates. To validate this approach, 15 commercially available solvents derived from glycerol have been confronted with nitrocellulose.

\section{Specifications and performance function}

To build up an overall performance function Perf suitable for evaluating the fitness of each candidate with respect to the $n_{\mathrm{p}}$ target properties $p$, the function defined by eqn (1) can be used

$$
\text { Perf }=\sum_{p=1}^{n_{\mathrm{p}}} w_{\mathrm{p}} \cdot F_{\mathrm{p}} / \sum_{p=1}^{n_{\mathrm{p}}} w_{\mathrm{p}}
$$

where $w_{\mathrm{p}}$ is the weight of each individual property and $F_{\mathrm{p}}$ is the individual performance function for the property $p$. The relevant properties $p$ that will drive solvent selection have to be defined. In the case study of the present work - solvent for nitrocellulose coating - the main properties for the solvent are (i) good solubilising power for nitrocellulose (ii) non-flammability and convenient evaporation profile to ensure correct film quality (iii) acceptable EHS profile. For each of these characteristics, a calculable property has to be defined and a target value should be chosen, according to functionality (solubilisation, evaporation) or regulatory constraints (flammability, EHS indexes). The properties calculated are the ones presented in Table 1 along with their weight in the total performance Perf (eqn (1)).

Their selections are clarified below. Fig. 2 shows the evolution of the associated individual performance functions $F_{\mathrm{p}}$, described by a Gaussian-type function (eqn (2)).

$$
F_{\mathrm{P}}(x)=\exp \left[\ln (\text { Val }) \cdot\left(\frac{\text { Prop }-x}{\text { Tol }}\right)^{2}\right]
$$

where Prop is the target property value, whereas Tol and Val are respectively the tolerance and the property performance value at tolerance. Those values are reported in Table 1 for each of the selected properties. Other functions like desirability functions could also be used. ${ }^{13}$
Table 1 Properties $p$ predicted by IBSS, which are used for calculating the individual $\left(F_{\mathrm{p}}\right)$ and the overall (Perf) performance functions. Prop, Tol, Val and $w_{p}$ are respectively the symbols for the target value, the tolerance, the performance at tolerance and the weight that appear in eqn

\begin{tabular}{|c|c|c|c|c|c|}
\hline Property, $p$ & Prop & $\begin{array}{l}\text { Prediction } \\
\text { method }\end{array}$ & Tol & Val & $w_{\mathrm{p}}$ \\
\hline \multicolumn{6}{|l|}{ Solubilisation } \\
\hline RED (Hansen approach) & 0 & MB2010 24 & 0.2 & 0.8 & 4 \\
\hline \multicolumn{6}{|l|}{ Volatility/flammability } \\
\hline Boiling point & $100-300^{\circ} \mathrm{C}$ & MG2001 25 & 3 & 0.8 & 2 \\
\hline Flash point & $>60^{\circ} \mathrm{C}$ & CPN2006 ${ }^{26}$ & 3 & 0.8 & 2 \\
\hline \multicolumn{6}{|l|}{ Environmental and safety } \\
\hline Toxicity $\left(\mathrm{LC}_{50}\right)$ & $<3$ & MY2001 27 & 1 & 0.8 & 0.5 \\
\hline $\begin{array}{l}\text { Biodegradability } \\
\left(\log K_{\text {ow }}\right)\end{array}$ & $<3$ & MG2002 28 & 0.3 & 0.8 & 0.5 \\
\hline Bioaccumulation (BCF) & $<100$ & VK1975 29,30 & 1 & 0.8 & 0.5 \\
\hline
\end{tabular}
(1) and (2)
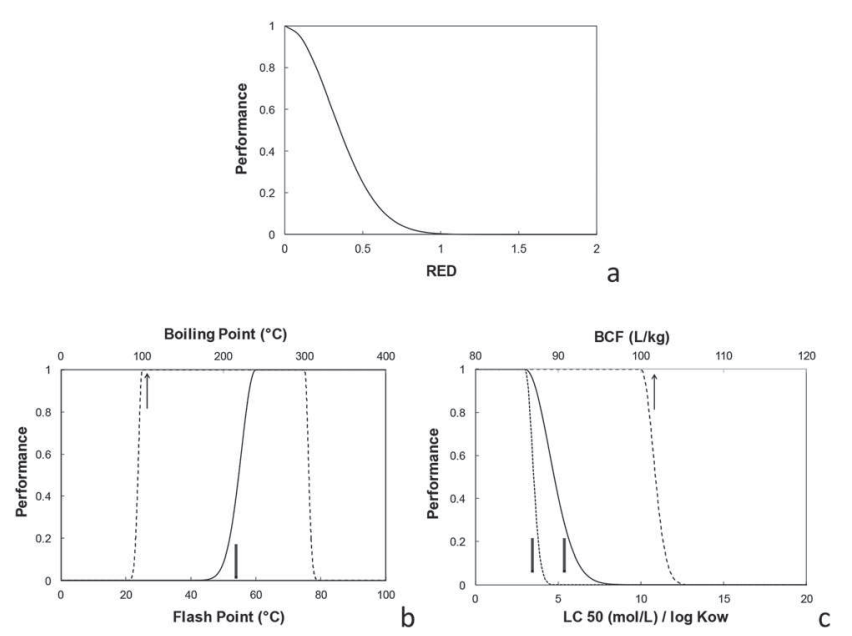

Fig. 2 Gaussian-type performance functions for properties listed in Table 1. (a) Solubilising power of a solvent expressed as its normalized distance from the center of the Hansen solubility sphere of nitrocellulose (b) solvent boiling point and flash point and (c) EHS descriptors for toxicity $\left(\mathrm{LC}_{50}\right)$, biodegradability (log $\left.K_{\mathrm{ow}}\right)$ and bioaccumulation (BCF) of solvents.

The ability to solubilise nitrocellulose is evaluated using the Hansen approach. ${ }^{24}$ The three Hansen solubility parameters, $\delta_{\mathrm{p}}, \delta_{\mathrm{h}}$ and $\delta_{\mathrm{d}}$, define a three-dimensional space in which all solvents and solutes can be located. ${ }^{10,31}$ A solute can be visualized as a point surrounded by its solubility sphere having a $R$ radius. All solvents and mixtures of solvents located inside this volume are likely to solubilise the solute. The closer the solute and solvents parameters are, the higher the solubility should be. The solute-solvent distance $D$ in the Hansen space is defined according to eqn (3).

$D=\sqrt{4\left(\delta_{d_{\text {solvent }}}-\delta_{d_{\text {solute }}}\right)^{2}+\left(\delta_{p_{\text {solvent }}}-\delta_{p_{\text {solute }}}\right)^{2}+\left(\delta_{h_{\text {solvent }}}-\delta_{h_{\text {solute }}}\right)^{2}}$

The ratio between the distance $D$ and the radius $R$ of the solubility sphere is called the "Relative Energy Difference" 
$(\operatorname{RED}=R / D)$ and allows a fast screening of molecules. This is the property calculated by IBSS to evaluate the solubilising capacities of the structures generated. RED $<1$ indicates that a molecule is inside the sphere and is likely to have a high affinity with the solute while higher values of RED indicate a poor affinity. The closer to 0 the RED value is, the better the solvent should be, that is why the target value for this property is set to 0 . Nitrocellulose has already been experimentally characterized by Hansen by testing its behaviour towards 88 classical solvents. ${ }^{24}$ Here, a grade of nitrocellulose, with $13.42 \%$ $\mathrm{N}$ and a MW of $69000 \mathrm{~g} \mathrm{~mol}^{-1}$ is investigated. From the experimental solubility data determined with conventional solvents we inferred a centre and a radius of the solubility sphere of the polymer, given in Table 2 and used for the calculation of RED.

The convenient evaporation profile is evaluated through the boiling point value. The solvent should be able to evaporate, but not too quickly to avoid formation of cracks in the nitrocellulose film. ${ }^{32}$ The higher limit has been set to $300{ }^{\circ} \mathrm{C}$, which may appear a bit high but is frequently encountered for high boiling point solvents used as coalescing agents for paints (dimethyl phthalate: $282{ }^{\circ} \mathrm{C}$, di(ethylene glycol) hexyl ether: $260{ }^{\circ} \mathrm{C}$, dimethyl isosorbide: $234^{\circ} \mathrm{C}$ ). The lower limit has been set to $100^{\circ} \mathrm{C}$, since toluene, which is currently used in lacquer thinners, has a boiling point of $111^{\circ} \mathrm{C}$. The non-flammability criterion is handled by calculating the flash point and setting a target value higher than $60{ }^{\circ} \mathrm{C}$ which is the lower limit for a compound to be classified as non-flammable.

Finally, toxicity is evaluated by setting a limit value for the $96 \mathrm{~h}$ fathead minnow acute toxicity $-\log _{10} \mathrm{LC}_{50}\left(\right.$ in $\mathrm{mol} \mathrm{L}^{-1}$ ) lower than 3 . This selected target value corresponds to $\mathrm{LC}_{50}>$ $100 \mathrm{mg} \mathrm{\textrm {L } ^ { - 1 }}$ for a molecule weighting $100 \mathrm{~g} \mathrm{~mol}^{-1}$, labelled as category 4 i.e. not harmful for aquatic environment in the United Nation Global Harmonization System of Hazard Classification and Labeling. ${ }^{29}$ Biodegradability is evaluated from the $K_{\text {ow }}$ octanol-water partition coefficient. Nonpolar compounds usually have values of $\log K_{\mathrm{ow}}>4$ and polar compounds have values of $\log K_{\mathrm{ow}}<1.5$. A target value $\log K_{\mathrm{ow}}<3$ is set. As regards to the Bioconcentration factor $\mathrm{BCF}$, a value greater than $500 \mathrm{~L} \mathrm{~kg}^{-1}$ indicates potential bioaccumulation. It is often correlated to the $K_{\mathrm{ow}}$ value. $^{29}$ Typically, a value of $3<\log K_{\mathrm{ow}}<4$ corresponds to $100<\mathrm{BCF}<500$. A target value $\mathrm{BCF}<100$ is set.

The potential solvent structures generated are compared and ranked using the performance function (eqn (1)) that takes into account the calculated RED, boiling point, flash

Table 2 Position of nitrocellulose in the Hansen space (experimental centre and radius of the sphere expressed in $\mathrm{MPa}^{1 / 2}$ )

\begin{tabular}{llllll}
\hline Nitrocellulose $\mathrm{e}^{a}$ & $\delta_{\mathrm{d}}$ & $\delta_{\mathrm{p}}$ & $\delta_{\mathrm{h}}$ & $R$ \\
\hline & 18.4 & 14.4 & 11.0 & 8.3 \\
& & & & \\
\end{tabular}

${ }^{a} \mathrm{MW}=69000 \mathrm{~g} \mathrm{~mol}^{-1}$ and $13.42 \% \mathrm{~N}$. point and the EHS indexes. It should also be pointed that a molecular weight limit has been set to $250 \mathrm{~g} \mathrm{~mol}^{-1}$ because "small" molecules are more likely to be liquid. Indeed, no melting point calculations have been performed since this property is poorly predicted by current methods, the average error being greater than $16^{\circ} \mathrm{C} .^{33}$

\section{Generation in silico of synthetically feasible "lead compounds" derived from glycerol with GRASS software}

In this paper, GRASS was deliberately stopped at the first generation to illustrate the methodology and to avoid combinatorial explosion. Solvents are commodity chemicals that should be obtained in a limited number of synthetic steps. $2^{\text {nd }}$ or $3^{\text {rd }}$ generation derivatives could also be considered, however, in the present paper, GRASS is used only as a proof of concept to generate synthetically feasible "lead compounds" further optimized by the IBSS program. Glycerol only possesses two primary and one secondary alcohol functional groups, therefore only 15 transformations, summarized in Table 3, among the initial 53 encoded in GRASS, ${ }^{11}$ are relevant to transform the glycerol molecule.

The industrial transformations encoded in GRASS allow the starting building block (glycerol in the present case) to react with implicit or explicit co-reactants, listed in Table 4. Implicit co-reactants are already encoded in the transformations

Table 3 Transformations of alcohols encoded in the GRASS program (numbers refer to those listed in Moity et al., 2014 ${ }^{11}$ )

\begin{tabular}{|c|c|c|c|c|}
\hline $\mathrm{Nb}$. & Alcohol & Co-reactant & $\begin{array}{l}\text { Final } \\
\text { function }\end{array}$ & $\begin{array}{l}\text { Name of } \\
\text { transformation }\end{array}$ \\
\hline $\mathrm{T} 1$ & $\doteq \mathrm{c}-\mathrm{OH}$ & & & Esterification \\
\hline T8 & $\searrow_{\mathrm{C}-\mathrm{OH}}$ & ${ }_{\mathrm{H}}^{\mathrm{H}} \mathrm{C}=\mathrm{C}_{\mathrm{H}}^{\mathrm{H}}$ & $\underset{\mathrm{H}_{2} \mathrm{C}-\stackrel{\mathrm{C}}{\mathrm{C}}-\mathrm{C} \mathrm{H}_{2}}{\stackrel{\mathrm{C}}{\leq}=}$ & Hydro-alkoxy -addition \\
\hline $\mathrm{T} 10$ & $\begin{array}{l}\mathrm{OH} \\
\mathrm{C}\end{array}$ & & & Acetalization \\
\hline T14 & 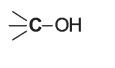 & $\mathrm{H}_{2} \mathrm{C}^{\prime}-\mathrm{CH}_{2}$ & $\begin{array}{l}\mathrm{HO} O-\mathrm{O} \\
\mathrm{H}_{2} \mathrm{C}-{ }_{\mathrm{CH}}\end{array}$ & Alcoholysis \\
\hline $\mathrm{T} 21$ & 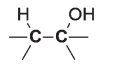 & $-\mathrm{H}_{2} \mathrm{O}$ & & Dehydration \\
\hline T32 & $\stackrel{\mathrm{H}}{\mathrm{H}} \mathrm{C}-\mathrm{OH}$ & $\mathrm{O}_{2}$ & & Oxidation \\
\hline T33 & $\mathrm{H}_{j}^{\prime} \mathrm{C}-\mathrm{OH}$ & $\mathrm{O}_{2}$ & & Oxidation \\
\hline $\mathrm{T} 40$ & $\begin{array}{l}\mathrm{OH} \\
\stackrel{\mathrm{l}}{\mathrm{C}}+\underset{\mathrm{OH}}{\mathrm{C}} \\
\mathrm{C}\end{array}$ & $\mathrm{CO}_{2}$ & & Carbonation \\
\hline $\mathrm{T} 41$ & $\doteq \mathrm{c}-\mathrm{OH}$ & $\mathrm{NH}_{3}$ & $\mathrm{NH}_{2}$ & Amino-de-hydroxylation \\
\hline $\mathrm{T} 42$ & ${ }_{\mathrm{C}-\mathrm{OH}}$ & $H N_{R}^{-R^{\prime}}$ & $N_{R}^{-R^{\prime}}$ & Amino-de-hydroxylation \\
\hline $\mathrm{T} 43$ & $\doteq \mathrm{C}-\mathrm{OH}$ & $\mathrm{R}-\mathrm{OH}$ & $-R$ & Alkoxy-de-hydroxylation \\
\hline T52 & $\doteq \mathrm{C}-\mathrm{OH}$ & $\mathrm{CO}$ & & Alcohol carbonylation \\
\hline T54 & $\doteq \mathrm{C}-\mathrm{OH}$ & $\mathrm{SO}_{3}$ & $-\mathrm{O}-\mathrm{SO}_{3} \mathrm{H}$ & Alcohol sulfation \\
\hline T56 & $\ni \mathrm{C}-\mathrm{OH}$ & $R_{1}^{\prime}$ & & Hemiacetalization \\
\hline T58 & $\underset{-\mathrm{CO}-\mathrm{C} i}{\mathrm{OH}}$ & Oxidant & - & Glycol oxidative cleavage \\
\hline
\end{tabular}


Table 4 Co-reactants chosen for the generation of "lead compounds" of glycerol-derivatives with the GRASS program

Explicit coreactants Implicit coreactants

Methyl amine $\left(\mathrm{R}=\mathrm{H}, \mathrm{R}^{\prime}=\mathrm{Me}\right)$, methanol $(\mathrm{R}=\mathrm{Me})$ acetic acid $(\mathrm{R}=\mathrm{Me})$, acetone $\left(\mathrm{R}=\mathrm{R}^{\prime}=\mathrm{Me}\right)$ $\mathrm{H}_{2}, \mathrm{H}_{2} \mathrm{O}, \mathrm{H}_{2} \mathrm{O}_{2}, \mathrm{CO}, \mathrm{CO}_{2}, \mathrm{NH}_{3}, \mathrm{O}_{2}, \mathrm{O}_{3}, \mathrm{SO}_{3}$, ethylene, ethylene oxide, formaldehyde

whereas explicit ones need to be provided to the program, as described in the original paper. ${ }^{11}$ The explicit co-reactants chosen are the most common monofunctional derivatives of the amine, alcohol, carboxylic acid and ketone chemical families that are reacted with the starting building block. This need to select only one representative member of each chemical family necessarily restrains the derivatives provided by GRASS. Other cheap and readily available co-reactants could have been selected in several families (e.g. ethanol in place of methanol, benzaldehyde in place of acetone, etc...), leading to other $1^{\text {st }}$ generation derivatives. That is why the compounds provided by GRASS should be regarded only as generic compounds to be further optimized by changing $\mathrm{R}$ and $\mathrm{R}^{\prime}$ groups in the IBSS program.

At the first generation, 66 molecules with a molecular weight lower than $250 \mathrm{~g} \mathrm{~mol}^{-1}$ are obtained (Table 5). On one hand, implicit co-reactants react with glycerol according to transformations of Table 3 giving: the oxidation products (T32, $\mathrm{T} 33$, T58), the dehydration products (T21), the products of amino-de-hydroxylation (T41), carbonylation (T52) and sulfation (T54). Addition of one, two or three of the hydroxyl group of glycerol on the double bond (T11) provides the five compounds listed for the reaction with ethylene. Carbonation with carbon dioxide (T40) gives the two cyclic carbonates. Ethylene oxide opening (T14) provides the five products of the reaction of one, two or three of the hydroxyl group of glycerol.

On the other hand, the explicit co-reactant methyl amine reacts through amino-dehydroxylation (T42) on one, two or three of the hydroxyl group providing five products. The derivatives obtained by reaction with methanol are the ethers produced by alkoxy-dehydroxylation (T43) occurring on one, two or three of the hydroxyl group of glycerol. With the same co-reactant, acetalization (T10) involving formaldehyde as implicit co-reactant provides the acetals. Esterification with acetic acid on one, two or three of the hydroxyl groups (T1) provide the five esters. Reaction with acetone gives access to the cyclic ketals (T10) and hemiketals (T56). Among the 66 structures generated by GRASS, 58 have a CAS registry number, which means that GRASS provides indeed synthetically feasible chemical structures. The compounds may or may not be experimentally obtained starting from glycerol. Relevant references describing the preparation of the compounds starting from glycerol and the selected co-reactant (or straightforward derivatives) in reasonable yields are given in Table 5 together with the global performance calculated by IBSS for the target application.

The properties predicted by IBSS may be compared to the experimental values when they are available. For calculating the boiling points, IBSS uses the 3-level group contribution method described by Marrero and $\mathrm{Gani}^{25}$ that has a reported accuracy of $8 \mathrm{~K}$ over 1211 data points. Fig. 3 shows the correlation between experimental and predicted boiling points for the 88 solvents of Hansen's original list (white dots) ${ }^{24}$ and the 33 glycerol derivatives for which reliable boiling points are reported in the literature at normal or reduced pressure (black dots). In this latter case, the boiling point has been extrapolated to $760 \mathrm{~mm} \mathrm{Hg}$ using P-T nomograph. There are some noticeable discrepancies in the "traditional solvents" family, particularly for very polar solvents that are suspected to be highly structured, like dimethylformamide (DMF) or propylene carbonate. It should be stressed that Marrero and Gani's prediction method was mainly regressed over molecules issued from the oil \& gas industry that did not include much molecules from green feedstocks. Similar discrepancies are observed for the glycerol derivatives obtained by GRASS. Glycerol itself is fairly well-predicted $\left(264^{\circ} \mathrm{C}\right.$ vs. $\left.288^{\circ} \mathrm{C}\right)$ but the boiling points of glycerol carbonate, glycerol formal or glycidol are clearly underestimated, probably because of the strong inter- and intra-hydrogen bonding. For other compounds, particularly the oxidation products, the discrepancy could be due to the wrong experimental determination of high boiling points for temperature-sensitive products. Nevertheless, the overall correlation can be considered as good, which means that IBSS provides an accurate prediction of the boiling points. Regarding the other physical data calculated, not enough experimental data are available for the glycerol derivatives to draw such correlations. The ability of 15 commercial glycerolderivatives to dissolve nitrocellulose has been evaluated experimentally (see last section) and validates the prediction of the solubilisation performance.

At this first generation, only 7 out of the 66 structures designed by GRASS have a global performance higher than $60 \%$. The best structure shows a performance of $69.4 \%$. It is the diamine resulting from the reaction of glycerol with methyl amine on the two primary positions. This compound is described in the literature (CAS number 83804-96-0, boiling point $304{ }^{\circ} \mathrm{C}$ ), but has not been obtained from glycerol so far and a straightforward and selective synthesis from glycerol is unlikely. The position of substitution and the number of amine substituents impact the performance since the other compounds obtained with methyl amine as co-reactant have performances lower than $60 \%$.

Other high performers are ethers, esters and acetals in which one alcohol function remains free. None of these compounds is reported to be easily prepared from glycerol in the literature. It is interesting to note that the 1,3-glycerol diethyl ether obtained by addition of ethylene has a higher ranking than the 1,3-glycerol dimethyl ether resulting from reaction with methanol, which tends to show that the length of the lateral chain impacts the performance. At this point, it is also important to stress that all the molecules obtained by virtually reacting glycerol with ethylene can be found in the literature, but they are not experimentally prepared starting from these two reactants. Instead, these ethers are obtained by reaction of glycerol with ethanol, another representative member of the 
Table 5 Performances of the 66 structures with $\mathrm{MW}<250 \mathrm{~g} \mathrm{~mol}^{-1}$ obtained as first generation derivatives of glycerol using the GRASS software. The co-reagents from which they were obtained are indicated: $\mathrm{H}_{2} \mathrm{CO}=$ formaldehyde, Ethy = ethylene, EtOx = ethylene oxide, $\mathrm{MA}=$ methyl amine, $\mathrm{MeOH}=$ methanol, $\mathrm{AcOH}=$ acetic acid, Acet = acetone and Ox = oxidant. Relevant references describing an efficient synthetic pathway from glycerol and the given co-reagent are indicated. Compounds without CAS registry number are indicated in italics

\begin{tabular}{|c|c|c|c|c|c|c|c|c|}
\hline Compound & Co-reagent & Perf \% & Compound & Co-reagent & Perf\% & Compound & Co-reagent & Perf \% \\
\hline $\mathrm{OH}$ & $\mathrm{H}_{2} \mathrm{CO}^{38}$ & 58.0 & & $\mathrm{H}_{2} \mathrm{CO}^{38}$ & 57.9 & & $\mathrm{NH}_{3}$ & 57.9 \\
\hline & $\mathrm{NH}_{3}{ }^{39}$ & 57.9 & & - & 57.9 & & $\mathrm{NH}_{3}$ & 57.9 \\
\hline & $\mathrm{NH}_{3}$ & 57.9 & & $\mathrm{NH}_{3}{ }^{40}$ & 57.9 & & $\mathrm{O}_{2}$ & 57.9 \\
\hline & $\mathrm{O}_{2}^{41}$ & 57.9 & & $\mathrm{O}_{2}$ & 57.9 & & $\mathrm{O}_{2}^{42}$ & 57.9 \\
\hline & $\mathrm{O}_{2}$ & 57.9 & & $\mathrm{O}_{2}$ & 57.9 & & - & 57.9 \\
\hline & $O x^{43}$ & 57.9 & & $\mathrm{O}_{2}$ & 53.4 & & $\mathrm{O}_{2}$ & 52.8 \\
\hline 0 & - & 50.0 & & - & 36.9 & & $\mathrm{CO}$ & 34.8 \\
\hline & $\mathrm{CO}$ & 34.8 & & $\mathrm{O}_{2}$ & 32.9 & & $\mathrm{SO}_{3}$ & 33.3 \\
\hline & $\mathrm{SO}_{3}$ & 33.3 & & $\mathrm{CO}$ & 31.6 & & - & 25.9 \\
\hline $\mathrm{O} \approx$ & Ox & 17.3 & & Ethy & 62.9 & & Ethy & 60.8 \\
\hline & Ethy & 59.5 & & Ethy & 58.0 & & Ethy & 57.4 \\
\hline & $\mathrm{CO}_{2}{ }^{44}$ & 57.9 & & $\mathrm{CO}_{2}^{45}$ & 57.9 & & EtOx & 58.0 \\
\hline & EtOx & 57.9 & & EtOx & 45.5 & & EtOx & 40.5 \\
\hline & EtOx & 38.5 & & MA & 69.4 & & $M A$ & 59.7 \\
\hline & MA & 58.6 & & MA & 58.6 & & MA & 58.4 \\
\hline & $\mathrm{MeOH}$ & 65.7 & & $\mathrm{MeOH}$ & 62.2 & & $\mathrm{MeOH}^{46}$ & 60.6 \\
\hline & $\mathrm{MeOH}^{47}$ & 59.7 & & $\mathrm{MeOH}$ & 59.7 & & $\mathrm{MeOH}$ & 59.1 \\
\hline & $\mathrm{MeOH}^{48}$ & 58.3 & & $\mathrm{MeOH}$ & 57.9 & & $\mathrm{MeOH}^{49}$ & 57.9 \\
\hline & $\mathrm{MeOH}^{50}$ & 39.5 & & $\mathrm{AcOH}$ & 60.9 & & $\mathrm{AcOH}^{51}$ & 59.9 \\
\hline & $\mathrm{AcOH}^{52}$ & 58.6 & & $\mathrm{AcOH}^{53}$ & 57.9 & & $\mathrm{AcOH}^{54}$ & 54.6 \\
\hline & Acet $^{55}$ & 58.4 & & Acet $^{56}$ & 58.5 & & Acet & 57.9 \\
\hline & Acet & 57.9 & $\mathrm{HO}$ & Acet & 57.9 & $\mathrm{HO}$ & Acet & 57.9 \\
\hline
\end{tabular}

monoalcohol chemical family that could have been chosen in place of methanol.

A second generation with the same co-reactants provides $c a$. 15000 GRASS derivatives, 2675 with a molecular weight lower than $250 \mathrm{~g} \mathrm{~mol}^{-1}$. This generation and the further ones have not been considered. Instead, the most promising families of GRASS compounds obtained at the first generation have been implemented to the IBSS database as complex functional 


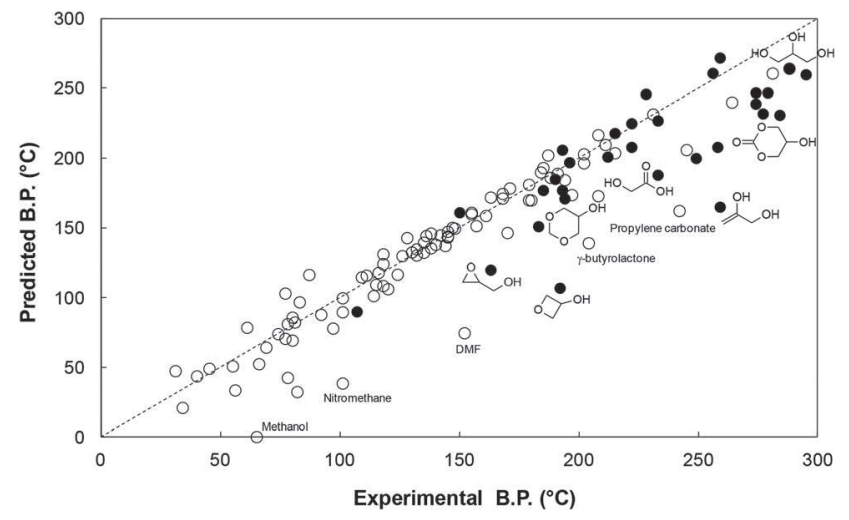

Fig. 3 Comparison between the boiling points predicted by IBSS and the experimental values reported for 88 classical solvents of Hansen's list (white dots) and glycerol-based solvents from the first GRASS generation (black dots).

groups to perform slight structural modifications of the substituents allowing a performance increase.

\section{Evolutionary structure optimisation by IBSS}

IBSS has been used to find the R and $\mathrm{R}^{\prime}$ substituents that most increase performance of the GRASS generic compounds shown in the first column of Table 6. These "lead compounds" have been obtained by reaction of glycerol with derivatives of the explicit GRASS co-reactant, i.e. 1-monoamines; mono-, di- and tri-ethers; 1-mono, 1,3-di and tri-esters and the C5- and C6ketals (see Fig. 4 and Table 6). IBSS has been asked to search for R substituents among linear or branched alkane, alkene, cycloalkane, cycloalkene and phenyl groups. The maximum number of carbon atoms has been set to 6 in order to limit the molecular weights of the compounds generated.

IBSS provides 92 new compounds with performances higher than the starting GRASS derivatives. A bibliographic search highlighted the compounds that have already been prepared from glycerol or a simple derivative in reasonable or high yields. These are not necessarily the best candidates found by IBSS. Fig. 4 illustrates the performance increase obtained thanks to IBSS structure optimization. For each generic family, 3 compounds are shown: the starting GRASS compound, the best IBSS candidate already described in the literature and the very best IBSS candidate. Table 6 shows the structures of these compounds.

It is worth to notice that a RED value below unity is already calculated for 10 out of the 11 GRASS first generation derivatives in Fig. 4, which means that they are themselves supposed to be good solvents of nitrocellulose. The global performance of these first generation compounds ranges from $39.5 \%$ to $60.6 \%$. The worst score of $39.5 \%$ is for glycerol trimethyl ether whereas glycerol mono- and di-methyl ethers score between $57.9 \%$ and $60.6 \%$, the best candidate being glycerol-1,3-dimethyl ether. Notice that the glycerol acetals also show high performances, with $58.5 \%$ for the 6-membered ring isomer (1,3-dioxane-type) and $58.4 \%$ for the 5 -membered ring one (dioxolane-type). These
Table 6 Results of the structure optimization by IBSS. For each family, the GRASS lead compounds and co-reagent are indicated as generic compounds. The performance are given for various substituents: the first line corresponds to the GRASS compound, the second one to the best IBSS compound found in the literature and the last line to the very best structure proposed by IBSS even when it is not known yet. Relevant references describing an efficient synthetic pathway are also indicated

\begin{tabular}{|c|c|c|c|c|c|}
\hline \multirow[b]{2}{*}{ Lead compounds } & \multirow[b]{2}{*}{ Co-reagent } & \multicolumn{2}{|c|}{ Substituents } & \multirow[b]{2}{*}{ Perf \% } & \multirow[b]{2}{*}{ Ref. } \\
\hline & & $\mathrm{R}$ & $\mathrm{R}^{\prime}$ & & \\
\hline \multirow{6}{*}{${ }^{-O} \sim O_{R}$} & \multirow[t]{3}{*}{$\mathrm{R}-\mathrm{OH}$} & $\mathrm{Me}$ & - & 39.5 & 51 \\
\hline & & Et & - & 57.4 & - \\
\hline & & - & - & - & - \\
\hline & \multirow{3}{*}{${ }_{\mathrm{RH}}^{\mathrm{O}}$} & $\mathrm{Me}$ & - & 54.6 & 55 \\
\hline & & $\mathrm{Et}$ & - & 54.9 & 58 \\
\hline & & 一 & - & - & - \\
\hline \multirow{2}{*}{ مل } & \multirow[t]{2}{*}{$\mathrm{R}-\mathrm{OH}$} & \multirow[t]{2}{*}{$\mathrm{Me}$} & - & 57.9 & 50 \\
\hline & & & - & 65.1 & 59 \\
\hline \multirow{3}{*}{$\underbrace{\mathrm{OH}} \mathrm{O}_{\mathrm{R}}$} & \multirow{3}{*}{$\mathrm{R}-\mathrm{OH}$} & & - & - & - \\
\hline & & \multirow{2}{*}{ 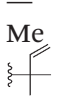 } & - & 58.3 & 49 \\
\hline & & & - & 61.5 & 60 \\
\hline & & $\xi$ & - & 62.5 & - \\
\hline & $\mathrm{R}-\mathrm{NH}$ & $\mathrm{H}$ & $\mathrm{Me}$ & 58.4 & - \\
\hline & & $\mathrm{H}$ & 5. & 61.6 & 61 \\
\hline & & $\mathrm{H}$ & हs & 67.1 & - \\
\hline & & $\mathrm{Me}$ & - & 58.6 & 53 \\
\hline & $\mathrm{OH}$ & & - & 64.1 & 62 \\
\hline & & & - & 69.1 & - \\
\hline & $\mathrm{R}-\mathrm{OH}$ & $\mathrm{Me}$ & $\mathrm{Me}$ & 59.1 & - \\
\hline & & Et & Et & 60.8 & 63 \\
\hline & & - & - & - & - \\
\hline & $\mathrm{R}-$ & $\mathrm{Me}$ & $\mathrm{Me}$ & 59.9 & 52 \\
\hline & $\mathrm{H}$ & 一 & 一 & - & - \\
\hline & & $\mathrm{Me}$ & Me & 58 & 57 \\
\hline & & $\mathrm{H}$ & $\xi$ & 61.7 & 64 \\
\hline & & & $\xi<$ & 71.8 & - \\
\hline & & Me & $\mathrm{Me}$ & 58.4 & 56 \\
\hline & & & $\xi$ & 63.5 & 65 \\
\hline & & $\xi$ & $\xi$ & 63.5 & - \\
\hline & $\mathrm{R}-\mathrm{OH}$ & $\mathrm{Me}$ & $\mathrm{Me}$ & 60.6 & 47 \\
\hline & & Et & Et & 62.9 & 20 \\
\hline & & - & - & - & - \\
\hline
\end{tabular}

latter compound is also known as solketal and is already used as coalescing agent in paints or lacquers. ${ }^{34}$

After IBSS optimization, three families rise out in terms of performance greater than 63\%: dioxane-type ketals (nine compounds with performance from $63.0 \%$ to $71.8 \%$ ) followed by glycerol esters (nine compounds with performances from $63.0 \%$ to $69.1 \%$ ) and then glycerol amines (six compounds with performances from $63.3 \%$ to $67.1 \%$ ). Then come glycerol2-methyl ether (65.1\% performance) and dioxolane-type ketals (three compounds with performances from $63.4 \%$ to $63.5 \%$ ). Several substituents improve significantly the performance for 


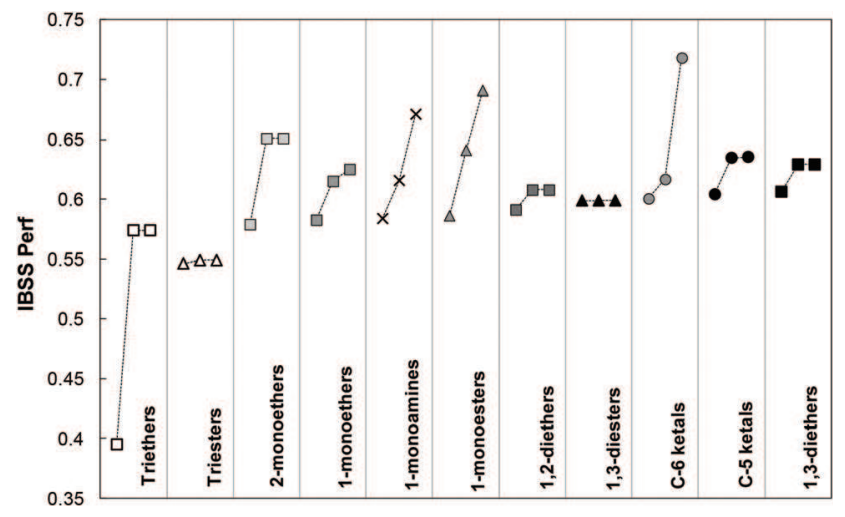

Fig. 4 Performance increase after structure optimization by IBSS. For each family, the first point corresponds to the GRASS derivative, the second one to the best structure proposed by IBSS for which a feasible synthetic pathway is described in the literature, and the third one to the very best structure proposed by IBSS. The structures are detailed in Table 6.

all compounds, especially isoalkyl and isoalkenyl isomers: 3-methylbut-1-ene, isopentyl, isobutyl and isohexyl isomers for monosubstituted compounds and the couple $\mathrm{R}=$ isopropyl, $\mathrm{R}^{\prime}$ = ethyl for disubstituted compounds. However, these nonsymmetrically disubstituted compounds were not retained since their straightforward preparation from glycerol is unlikely. On the other hand, cyclic substituents apart from 1-cyclopentenyl and apart from 2-cyclopentenyl are not favoured by IBSS to improve the performance.

Finally, it is interesting to stress that some of the top performers highlighted by IBSS can be easily accessed experimentally through catalytic routes (Fig. 5). Noticeably, the experimental preparation of the best esters and ethers do not take the GRASS pathway: the access to esters via the hydroalkoxycarbonylation of alkenes is encoded within GRASS (transformation 92 listed in reference ${ }^{11}$ ) but was not retained in the short list of industrially-relevant reactions; the preparation of ethers via the reductive alkylation of ketones has not been included in

$$
\text { (29\% yield, } 55 / 45 \text { mono/di }
$$

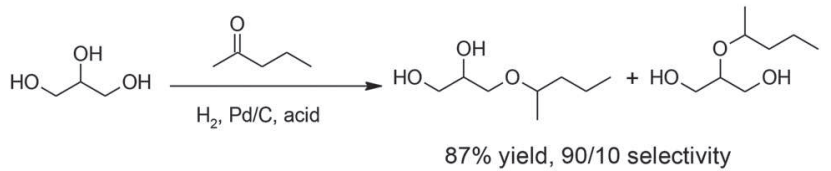

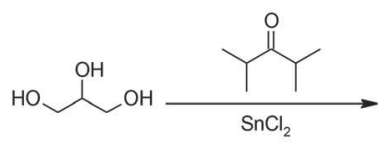

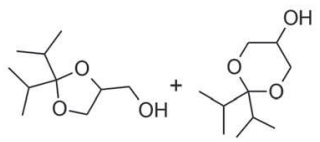

$42 \%$ yield, $78 / 22$ selectivity

Fig. 5 Experimental catalytic accesses to top performers in the esters ${ }^{36}$ ethers ${ }^{37}$ and ketals ${ }^{35}$ families. the list of GRASS transformations so far because it is not industrially applied yet. As discussed in an earlier publication, GRASS is a scalable software that allows adding further coreagents or chemical transformations newly published in the literature or in patents insofar as they seem applicable to the preparation of compounds on large scale. Interestingly, the best C5 and C6 ketals have recently been obtained by reaction of glycerol with diisopropylketone at room temperature and solvent-free conditions using a recyclable tin catalyst. ${ }^{35}$ The authors could prepare a series of ketals with varying ratios of 1,3-dioxane and 1,3-dioxolane isomers depending on the bulkiness of the starting ketones. As we showed in a recent publication devoted to glycerol acetals and ketals, one often gets the two isomers (dioxane and dioxolane) in varying proportions. It is difficult to obtain a single isomer but this is not an inconvenience because their solubilizing properties are very close. However their stability towards acid cleavage and oxidative degradation by oxygen are significantly different. ${ }^{23}$

The example described in this paper therefore proves the relevancy of combining GRASS and IBSS approaches to generate in silico synthetically feasible structures with predefined properties for a target application.

\section{Experimental validation of the approach with commercially available glycerol-derived solvents}

Among the list of the most promising solvents shown in Table 6, several compounds are commercially available. In particular the performance values of solketal, glycerol diacetates (two isomers) and 3-methylamino-1,2-propanediol are close to $60 \%$. Therefore, to validate the top-down approach described above, we have assessed the ability of these solvents to solubilize nitrocellulose and compared them to 11 other glycerolbased solvents. Table 7 summarizes the results obtained. Only 5 solvents effectively dissolve nitrocellulose (score 1 and 2)

Table 7 Experimentally determined solubility of nitrocellulose in 15 glycerol-derived solvents characterized by their Hansen solubility parameters $\left(\delta_{\mathrm{d}}, \delta_{\mathrm{p}}, \delta_{\mathrm{h}}\right)$ in $\mathrm{MPa}^{1 / 2}$. Solubility parameters were calculated thanks to the Yamamoto Molecular Break (Y-MB) group-contribution method (HSPiP software). The first column expresses our results of the $10 \% \mathrm{w} / \mathrm{v}$ solubility test using visual inspection as described in the experimental part: from 1 for completely soluble to 6 for no visible effect

\begin{tabular}{lllrrr}
\hline Glycerol derivative & Score & $\delta_{\mathrm{d}}$ & \multicolumn{1}{c}{$\delta_{\mathrm{p}}$} & $\delta_{\mathrm{h}}$ & RED \\
\hline 1,2-Diacetin & 2 & 16.5 & 14.3 & 16.3 & 0.79 \\
1,3-Diacetin & 2 & 16.4 & 7.5 & 13.1 & 0.99 \\
Tripropionin & 2 & 16.2 & 6.8 & 8.2 & 1.11 \\
Triacetin & 2 & 16.4 & 5.7 & 9.6 & 1.17 \\
3-Methylamino-1,2-propanediol & 1 & 16.2 & 11.6 & 19.2 & 1.17 \\
3-Ethoxy-1,2-propanediol & 6 & 16.1 & 8.7 & 15.9 & 1.06 \\
Glycerol formal & 6 & 18.3 & 10.1 & 19.9 & 1.19 \\
3-Methoxy-1,2-propanediol & 6 & 16.2 & 9.7 & 18.8 & 1.22 \\
Glycerol carbonate & 3 & 18.7 & 22.1 & 18.2 & 1.27 \\
Glycidol & 6 & 17.4 & 12.7 & 21.5 & 1.30 \\
3-tert-Butoxy-1,2-propanediol & 6 & 15.5 & 6 & 15.3 & 1.33 \\
3-Oxetanol & 3 & 18.3 & 11.5 & 21.8 & 1.35 \\
Solketal & 6 & 16.8 & 8.6 & 14.2 & 0.89 \\
$\alpha, \alpha^{\prime}$-Diglycerol & 6 & 16.9 & 11.6 & 25.3 & 1.79 \\
Glycerol & 6 & 17.4 & 11.3 & 27.2 & 2.00
\end{tabular}


whereas most of the others, including solketal, do not interact at all with this polymer (score 6). In principle, all solvents that have a RED value less than 1 should be good solvents for nitrocellulose (score 1 and 2). This is indeed the case for the diacetin isomers (RED $=0.79$ and 0.99 ) but not for solketal (RED = 0.89 ) which is an outlier. In the same way all solvents having a RED value higher than 1 are expected to be poor solvents. Actually most of these solvents have a score of 6 but there are three outliers (triacetin, tripropionin and 3-methylamino-1,2propanediol) which efficiently dissolve nitrocellulose (score 2).

Fig. 6 shows even more clearly the results of the solubilization experiments. Fig. $6 \mathrm{a}$ and $\mathrm{b}$ are the projections onto the $\left(\delta_{\mathrm{p}}, \delta_{\mathrm{h}}\right)$ and the $\left(\delta_{\mathrm{d}}, \delta_{\mathrm{h}}\right)$ planes respectively of the Hansen solubility sphere of nitrocellulose. The cross is the centre of the
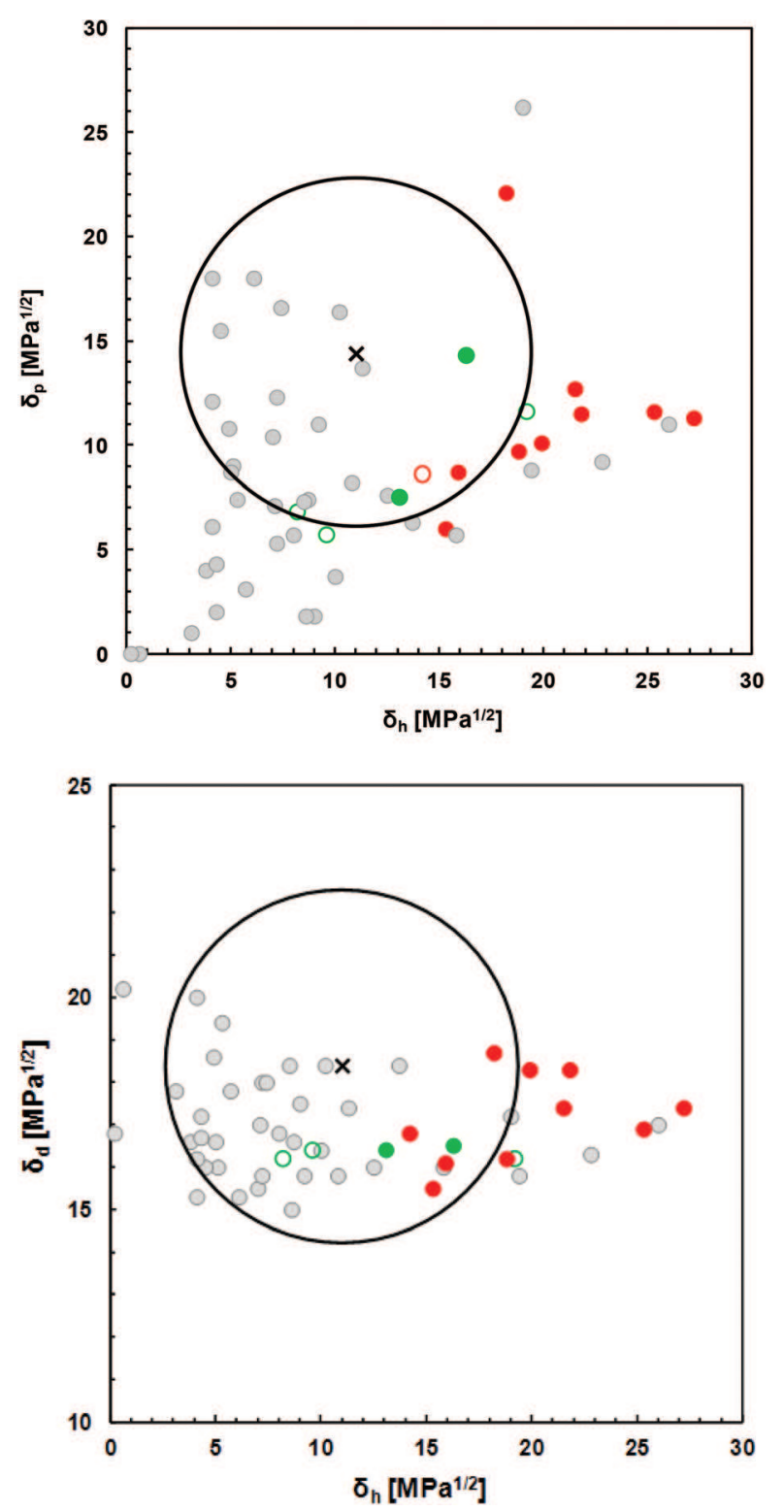

Fig. 6 Projections onto the $\left(\delta_{\mathrm{p}}, \delta_{\mathrm{h}}\right)$ and the $\left(\delta_{\mathrm{d}}, \delta_{\mathrm{h}}\right)$ planes of the Hansen's solubility sphere of nitrocellulose. sphere, grey dots represent conventional solvents used to build the sphere whereas red and green dots correspond to the poor and the effective glycerol-derived solvents respectively. Green circles, correspond to good solvents outside the sphere and the red circle to the sole bad solvent inside the sphere. It is noteworthy that these four outliers are close to the boundary between bad and good solvents. These results are in fair agreement with the predictions of IBSS and allow highlighting diacetin as an effective, inexpensive and non-hazardous solvent of nitrocellulose. Actually, commercial diacetin (B.P. $=240{ }^{\circ} \mathrm{C}, \mathrm{FP}$ $=110^{\circ} \mathrm{C}$, safe and unlabelled substance in the EU) is a mixture of the 1,2 and 1,3 isomers, which is inconsequential to the solubility efficiency since both of them are located within the Hansen's sphere of nitrocellulose.

\section{Conclusions}

Today, the art of organic synthesis has reached such a level of sophistication that chemists are able to synthesize almost any molecule with a given structure. The challenges of modern chemistry have therefore moved to other ambitious objectives including the design of molecules having predefined functional properties. The archetypical example is therapeutic chemistry which has to identify, among thousands of possible derivatives of a lead drug compound, the most effective one with regard to biological activity. These so-called fine chemicals are high value substances, produced in low volumes using multistep processes. The design of a bio-based solvent suitable for solubilising a precise target is derived from the same logic. However, due to the higher tonnages required and the auxiliary role of solvents, their synthesis must imperatively be green and simple. In contrast to the total synthesis of complex organic compounds for which numbers of advanced strategies have been developed, to the best of our knowledge, a systematic methodology for designing bio-based solvents suitable for a given application does not exist.

The coupling of two programs, namely GRASS and IBSS, provides an effective answer to this issue. In the first step, GRASS applies relevant chemical transformations to any starting building block to provide synthetically feasible molecular candidates. In the second step, IBSS generates hundreds of derivatives from these candidates, considered as lead compounds, and predicts their functional properties in order to highlight and rank the top performers which best fit the required specifications. To illustrate our approach we chose glycerol as the starting building block and nitrocellulose as the target solute. However the method can be generalized to any starting molecule and any molecular or macromolecular solute. Furthermore, for the sake of clarity, we limited GRASS to the first generation, while syntheses in two or three steps are quite acceptable even for commodity products such as solvents. Thanks to this limitation, we were able to discuss in detail each step of the process and search in the literature, candidates which are actually obtained from glycerol or one of its precursors. In a real situation, these two programs would gene- 
rate and sort, in a reasonable time, thousands of virtual compounds. Thus, GRASS would provide, in the third generation, many thousands of structures whose groups $\mathrm{R}$ and $\mathrm{R}^{\prime}$ would be exchanged by IBSS. During the last step, the values of the performance function $F_{\mathrm{p}}$ would be calculated automatically to allow the ranking of candidates according to their ability to meet specifications. Finally, a synthetic chemist should carefully examine the list of the most promising compounds in order to select those that seem the most easily accessible even if the synthesis has never been described before.

\section{Experimental}

\section{GRASS program}

GRASS is a Computer-Assisted Organic Synthesis program that derives from GRAAL, a software developed by Barone et al. to generate all possible degradation products of food flavours. ${ }^{62}$ The detailed description of the program architecture is given in the original papers. ${ }^{62,63}$ We have recently updated this software to generate systematically all plausible molecules from a given bio-based building block, according to well-chosen transformations and co-reactants. ${ }^{11}$ Particularly, the careful choice of 53 transformations on the basis of industrial relevancy ascertains that the structures generated are feasible. The input data are the starting building-block - glycerol in the present case - the list of transformations and the list of co-reactants. The outputs are the generated structures which are encoded by their SMILE code.

\section{IBSS program}

The IBSS program is a Computer Aided Product Design (CAPD) tool that was developed for finding sustainable products. ${ }^{13,64}$ The principle of the Computer Aided Molecular Design (CAMD) is to define initially a set of target values for several properties and search for new molecules built from chemical blocks, that satisfy the set of properties. ${ }^{65}$ The IBSS tool was expanded to search for mixtures and is able to orientate the search towards molecules issued from renewable material feedstocks. ${ }^{13}$ In the present work, single molecules are searched, each containing one of the core chemical fragments proposed by the GRASS program.

The problem to solve is then a multi-objective simultaneous search over the optimization variables like the molecules described by a molecular graph $\mathrm{MG}_{i}$, the mixture composition $z_{i}$ and the mixture operating conditions $\operatorname{cond}_{i}$. The performance of each candidate molecule or mixture is calculated from a performance function described above, which takes into account the calculated RED (distance from the centre of the Hansen solubility sphere of nitrocellulose over the radius of this sphere), boiling point, flash point and the EHS indexes mentioned before.

\section{Solubility of nitrocellulose in glycerol-based solvents}

For solubility tests, $100 \mathrm{mg}$ of nitrocellulose are placed in a $1.5 \mathrm{~mL}$ glass vial, and $1.0 \mathrm{~mL}$ of solvent is added. The mixture is stirred at $60 \mathrm{rpm}$ using a Intelli-Mixer RM-2L rotator (Elmi) during $24 \mathrm{~h}$ at $(25 \pm 1){ }^{\circ} \mathrm{C}$. The classification of solubility behaviours is based on visual observations: score 1 for soluble, 2 for almost soluble, 3 for strongly swollen but slightly soluble, 4 for swollen, 5 for little swelling and 6 for no visible effect. A solvent is considered as a good solvent when its score equals 1 or 2 .

For building the Hansen solubility sphere of nitrocellulose, 40 well-selected conventional solvents are confronted to nitrocellulose. ${ }^{10,31}$ Results were computed with the HSPiP software, version 4.0.05 developed by Abbott and Yamamoto, to calculate the solubility spheres coordinates - centre and radius - of nitrocellulose. Then 15 glycerol-based solvents were tested with the same procedure.

\section{Acknowledgements}

This work was performed within the framework of ANR "InBioSynSolv" project. The authors gratefully acknowledge the gift of nitrocellulose from Pr. Pierre Gareil (Ecole Nationale Supérieure de Chimie de Paris) and the help of R. Barone in designing the GRASS program.

\section{References}

1 C. Capello, U. Fischer and K. Hungerbühler, Green Chem., 2007, 9, 927-934.

2 P. G. Jessop, Green Chem., 2011, 13, 1391-1398.

3 F. M. Kerton and R. Marriott, Alternative Solvents for Green Chemistry, Royal Society of Chemistry, 2013.

4 L. Moity, M. Durand, A. Benazzouz, V. Molinier and J.-M. Aubry, in Alternative Solvents for Natural Products Extraction, ed. F. Chemat and M. A. Vian, Springer, Berlin Heidelberg, 2014, pp. 1-24.

5 A. Corma, S. Iborra and A. Velty, Chem. Rev., 2007, 107, 2411-2502.

6 J. J. Bozell and G. R. Petersen, Green Chem., 2010, 12, 539554.

7 R. A. Sheldon, Green Chem., 2014, 16, 950-963.

8 M. Durand, V. Molinier, W. Kunz and J.-M. Aubry, Chem. Eur. J., 2011, 17, 5155-5164.

9 L. Moity, M. Durand, A. Benazzouz, C. Pierlot, V. Molinier and J.-M. Aubry, Green Chem., 2012, 14, 1132-1145.

10 A. Benazzouz, L. Moity, C. Pierlot, V. Molinier and J.-M. Aubry, Colloids Surf., A, 2014, 458, 101-109.

11 L. Moity, V. Molinier, A. Benazzouz, R. Barone, P. Marion and J.-M. Aubry, Green Chem., 2014, 16, 146-160.

12 J. Heintz, I. Touche, M. Teles Dos Santos and V. Gerbaud, Comput. -Aided Chem. Eng., 2012, 30, 702-706.

13 J. Heintz, J.-P. Belaud, N. Pandya, M. Teles Dos Santos and V. Gerbaud, Comput. Chem. Eng., 2014, 71, 362-376.

14 M. Pagliaro, R. Ciriminna, H. Kimura, M. Rossi and C. Della Pina, Angew. Chem., Int. Ed., 2007, 46, 4434-4440.

15 A. Behr, J. Eilting, K. Irawadi, J. Leschinski and F. Lindner, Green Chem., 2008, 10, 13-30. 
16 C.-H. (Clayton) Zhou, J. N. Beltramini, Y.-X. Fan and G. Q. (Max) Lu, Chem. Soc. Rev., 2008, 37, 527-549.

17 H. Zhang and M. W. Grinstaff, Macromol. Rapid Commun., 2014, 35, 1906-1924.

18 A. E. Díaz-Álvarez, J. Francos, B. Lastra-Barreira, P. Crochet and V. Cadierno, Chem. Commun., 2011, 47, 6208.

19 Y. Gu and F. Jérôme, Green Chem., 2010, 12, 1127.

20 J. I. García, H. García-Marín, J. A. Mayoral and P. Pérez, Green Chem., 2010, 12, 426.

21 J. I. García, H. García-Marín and E. Pires, Green Chem., 2014, 16, 1007.

22 Y. Gu and F. Jérôme, Chem. Soc. Rev., 2013, 42, 9550-9570.

23 L. Moity, A. Benazzouz, V. Molinier, V. Nardello-Rataj, M. K. Elmkaddem, P. de Caro, S. Thiébaud-Roux, V. Gerbaud, P. Marion and J.-M. Aubry, Green Chem., 2015, 17, 1779-1792.

24 C. M. Hansen, Hansen Solubility Parameters: A User's Handbook, Second Edition, CRC Press, 2007.

25 J. Marrero and R. Gani, Fluid Phase Equilib., 2001, 183-184, 183-208.

26 L. Catoire, S. Paulmier and V. Naudet, Process Saf. Prog., 2006, 25, 33-39.

27 T. M. Martin and D. M. Young, Chem. Res. Toxicol., 2001, 14, 1378-1385.

28 J. Marrero and R. Gani, Ind. Eng. Chem. Res., 2002, 41, 6623-6633.

29 Structure-Activity Correlations in Studies of Toxicity and Bioconcentration with Aquatic Organisms, ed. G. D. Veith and D. E. Konasewich, Great Lakes Research Advisory Board, 1975.

30 M. Sinha, L. E. K. Achenie and G. M. Ostrovsky, Comput. Chem. Eng., 1999, 23, 1381-1394.

31 A. Benazzouz, L. Moity, C. Pierlot, M. Sergent, V. Molinier and J.-M. Aubry, Ind. Eng. Chem. Res., 2013, 52, 16585-16597.

32 M. Durand, V. Molinier, T. Féron and J.-M. Aubry, Prog. Org. Coat., 2010, 69, 344-351.

33 A. S. Hukkerikar, B. Sarup, A. Ten Kate, J. Abildskov, G. Sin and R. Gani, Fluid Phase Equilib., 2012, 321, 25-43.

34 J. L. Couturier, J. P. Lallier and J. L. Dubois, Arkema France, Use of Glycerol Acetals as Coalescing Agents for Lacquers, Paints or Varnishes and Composition Thereof, WO 2011/042652 A1, 2011.

35 M. J. da Silva, M. de O. Guimaraes and A. A. Julio, Catal. Lett., 2015, 145, 769-776.

36 K. A. Suerbaev, E. G. Chepaikin, N. O. Appazov and B. Z. Dzhiembaev, Pet. Chem., 2012, 52, 189-193.

37 Y. Shi, W. Dayoub, G.-R. Chen and M. Lemaire, Sci. China: Chem., 2010, 53, 1953-1956.

38 V. R. Ruiz, A. Velty, L. L. Santos, A. Leyva-Pérez, M. J. Sabater, S. Iborra and A. Corma, J. Catal., 2010, 271, 351-357.

39 Y.-H. Joo and J. M. Shreeve, Chem. Commun., 2010, 46, 142144.

40 B. Andreeßen and A. Steinbüchel, Appl. Microbiol. Biotechnol., 2011, 93, 357-365.

41 R. M. Painter, D. M. Pearson and R. M. Waymouth, Angew. Chem., Int. Ed., 2010, 49, 9456-9459.
42 M. Trincado, K. Kühlein and H. Grützmacher, Chem. - Eur. J., 2011, 17, 11905-11913.

43 M. Ziolek, I. Sobczak, P. Decyk and L. Wolski, Catal. Commun., 2013, 37, 85-91.

44 M. S. Khayoon and B. H. Hameed, Appl. Catal., A, 2013, 466, 272-281.

45 T. W. Turney, A. Patti, W. Gates, U. Shaheen and S. Kulasegaram, Green Chem., 2013, 15, 1925.

46 J.-J. Hu, Chin. Chem. Lett., 2013, 24, 1106-1108.

47 Y. Matsumoto, K. Mita, K. Hashimoto, H. Iio and T. Tokoroyama, Tetrahedron, 1996, 52, 9387-9398.

48 L. Birkofer and O. Stuhl, J. Organomet. Chem., 1980, 187, 21-29.

49 E. Quesada, J. Delgado, C. Gajate, F. Mollinedo, A. U. Acuña and F. Amat-Guerri, J. Med. Chem., 2004, 47, 5333-5335.

50 W. Dayoub, M. Sutter, E. Metay, Y. Raoul and M. Lemaire, ChemCatChem, 2013, 26, 5902-5916.

51 M. Zieringer, M. Wyszogrodzka, K. Biskup and R. Haag, New J. Chem., 2012, 36, 402-406.

52 R. Srivastava and N. Venkatathri, Indian J. Chem. Sect. B: Org. Chem. Incl. Med. Chem., 2004, 43, 888-891.

53 I. Dosuna-Rodríguez and E. M. Gaigneaux, Catal. Today, 2012, 195, 14-21.

54 J. Ye, S. Liu, J. Xiang, J. Lei and C. Zhou, J. Appl. Polym. Sci., 2013, 129, 1915-1921.

55 A. W. Pierpont, E. R. Batista, R. L. Martin, W. Chen, J. K. Kim, C. B. Hoyt, J. C. Gordon, R. Michalczyk, L. A. "Pete" Silks and R. Wu, ACS Catal., 2015, 5, 1013-1019.

56 B. Wang, Y. Shen, J. Sun, F. Xu and R. Sun, RSC Adv., 2014, 4, 18917.

57 R. Prager and Z. Yurui, Aust. J. Chem., 1989, 42, 1003-1005.

58 D. Güclü, M. Rale and W.-D. Fessner, Eur. J. Org. Chem., 2015, 2960-2964.

59 C. Bonini, L. Chiummiento, N. Di Blasio, M. Funicello, P. Lupattelli, F. Tramutola, F. Berti, A. Ostric, S. Miertus, V. Frecer and D.-X. Kong, Bioorg. Med. Chem., 2014, 22, 4792-4802.

60 S. Pariente, N. Tanchoux and F. Fajula, Green Chem., 2009, 11, 1256.

61 I. Notar Francesco, B. Cacciuttolo, M. Pucheault and S. Antoniotti, Green Chem., 2015, 17, 837-841.

62 R. Barone, C. Chanon, G. Vernin and C. Parkanyi, in Food Flavor and Chemistry; Explorations into the 21st Century, ed. A. M. Spanier, F. Shahidi, T. H. Parliment, C. Mussinan and E. Tratras Contis, RSC, Cambridge, UK, 2005 pp. 175212.

63 C.-T. Ho and E. T. Contis, Recent Advances in Food and Flavor Chemistry: Food Flavors and Encapsulation, Health Benefits, Analytical Methods, and Molecular Biology of Functional Foods, Royal Society of Chemistry, 2010.

64 J. Heintz, J.-P. Belaud and V. Gerbaud, Comput. Ind., 2014, 65, 505-520.

65 L. Achenie, V. Venkatasubramanian and R. Gani, Computer Aided Molecular Design: Theory and Practice, Elsevier, 2002. 